Title : will be set by the publisher

Editors : will be set by the publisher

EAS Publications Series, Vol. ?, 2006

\title{
PILOT - THE PATHFINDER FOR AN INTERNATIONAL LARGE OPTICAL TELESCOPE
}

\author{
J.W.V. Storey ${ }^{1}$, M.C.B. Ashley ${ }^{1}$, M.G. Burton ${ }^{1}$ and J.S. Lawrence ${ }^{1}$
}

\begin{abstract}
PILOT is proposed as a partnership between Australia and Europe to develop a 2.4 metre optical/infrared telescope for Dome C, Antarctica. Funding for a detailed designed study is being sought from Australian sources, with a view to commencing construction in early 2008. The current "strawman" design is for an $\mathrm{f} / 10$ dual Nasmyth configuration with provision for both a silicon carbide fast tip-tilt secondary mirror for the thermal infrared, and an adaptive secondary mirror to achieve diffraction-limited imaging at wavelengths as short as V-band.
\end{abstract}

\section{Introduction}

The Antarctic Plateau, including sites such as Dome C, offers a remarkable opportunity for astronomy. The intense cold, high altitude, and stable atmospheric conditions combine to promise sensitivities and image quality that surpass those found anywhere else on earth. Most importantly, an Antarctic telescope can outperform a similar one at a temperature location that is several times the diameter and hence very much more expensive. This implies that for many of the most compelling scientific investigations, an Antarctic telescope may deliver the most cost-effective solution. As the cost of new telescopes rises from the tens of millions of Euros into the hundreds of millions, it becomes even more important to investigate the "Antarctic option". One of the big unknowns, however, is the actual cost of constructing and operating a large optical telescope in Antarctica. This can only be determined by building intermediate-scale facilities, such as the proposed PILOT telescope.

PILOT - the Pathfinder for an International Large Optical Telescope - is proposed as an international collaboration, bringing together astronomers whose ultimate scientific goals are very diverse, but who recognise the need for a common

\footnotetext{
1 School of Physics, University of New South Wales, Sydney, NSW 2052 Australia; e-mail: j.storey@unsw.edu.au
} 
pathfinder project. In the present era of well-established 8-10 metre telescopes at temperate sites, it is difficult for a small telescope to find a competitive scientific niche. Nevertheless, as demonstrated by Burton et al. (2005), a 2.4 metre telescope at Dome $\mathrm{C}$ can achieve very important results across a wide range of scientific endeavour.

\section{Concordia Station}

The opening of the new French/Italian Concordia Station at Dome C has allowed the early predictions of Harper (1989) and Gillingham (1991) of exceptional observing conditions on the Antarctic Plateau to be further verified. Initial studies at the South Pole had shown that improvements of up to two orders of magnitude were possible in the infrared sky brightness. However, while the free-atmosphere seeing was found to be excellent, the South Pole itself suffered from an intense, turbulent surface layer some several hundred metres thick.

At Dome $\mathrm{C}$, the free atmosphere seeing is once again superb (Lawrence et al. 2004; Agabi et al. 2006) and, partly as a result of the lower surface wind speeds, the turbulent surface layer is only of order 30 metres thick. Atmospheric modelling by Swain \& Gallee (2006) suggests that the regions on the plateau where the surface layer are thin lie along a ridge extending from Dome $\mathrm{C}$ through Dome A to Dome F, although this has yet to be directly confirmed.

For the foreseeable future, Concordia Station is the best accessible site for opti$\mathrm{cal} /$ infrared astronomy in the world. It is important to fully explore the opportunities presented by this remarkable site on a time scale that allows full advantage to be taken of the site conditions.

\section{Past, present and future telescopes}

SPIREX, the South Pole Infrared EXplorer (Hereld 1994; Fowler et al. 1998), was operational at the South Pole during the second half of the 1990s. SPIREX demonstrated that the sensitivity gains derived from the site testing results were realisable in practice, and could be directly translated into scientific outcomes. Although only a modest $60 \mathrm{~cm}$ in diameter, SPIREX achieved a string of noteworthy successes, particularly in the last two seasons of operation when it was fitted with an InSb array camera.

At Dome C, the IRAIT project (Tosti et al., these proceedings) will be an important next step. Operating from a modified shipping container, this $80 \mathrm{~cm}$ telescope is designed to observe mainly in the mid-infrared. Lessons learned from its deployment and operation will be of the utmost importance to PILOT.

The main purpose of PILOT is to act as a "pathfinder" and to open up opportunities for a wide range of more ambitious facilities. This might include interferometers, very-wide-field survey telescopes, and large, high resolution filled-aperture telescopes such as LAPCAT (Storey et al. 2006). 


\section{Is $2.4 \mathrm{~m}$ the optimum size?}

PILOT must be large enough that it demonstrates, through important astronomical discoveries, that the advantages mooted for Dome $\mathrm{C}$ are realisable across a broad range of scientific problems. It must be large enough to explore natural seeing that drops below 0.1 arcseconds, and for this a diameter of at least 2 metres is desirable. Several of the science goals in the $2.3 \mu \mathrm{m}$ "cosmological window" require image sizes of order 0.2 arcseconds, again implying a $\sim 2.5$ metre aperture. At wavelengths as short as $900 \mathrm{~nm}$, simple tip-tilt correction should allow neardiffraction-limited imaging on this size telescope for a significant fraction of the time. Beyond $3.5 \mu \mathrm{m}$, a 2.4 metre telescope is expected to be diffraction-limited for almost all the time at Dome $\mathrm{C}$, even without tip-tilt. Under these conditions the point-source sensitivity is proportional to the square of the telescope diameter, imposing a large penalty on smaller telescopes for this type of science.

On the other hand, a 2.4 metre telescope is about the largest practical size for a pathfinder, for a number of important reasons:

- Telescopes of this size are in common production and are commercially available, making them relatively inexpensive. There is a large jump in price for larger telescopes.

- A 2.4 m telescope can make use of simple technologies such as a passive mirror support, thus reducing the technical risk of adapting them to Antarctic conditions.

- The size of all of the optical components in the instruments scales as $A \Omega$, where $A$ is the area of the telescope aperture and $\Omega$ the field-of-view. The cost of each of the instruments (not including detectors) for a telescope therefore scales as at least the telescope diameter squared.

- A $2.4 \mathrm{~m}$ telescope is fairly straightforward to transport in standard containers.

- The telescope enclosure is of modest dimensions.

It is intended that PILOT will be capable of both wide-field imaging (up to about 30 arcminute diameter) and high-angular resolution imaging across a narrow field-of-view in the visible. The latter could be achievable with an adapative secondary mirror of the type developed at Arcetri Observatory.

\section{Towers}

Before the height of the turbulent surface layer was known, it was hoped that a small snow hill could be built to place the telescope into the free air. However, if the surface layer is about $30 \mathrm{~m}$ thick, as appears to be the case, it is probably more practical to put the telescope on top of a steel tower. Solar telescopes are 
often placed on similarly-sized towers for exactly the same reason, as the daytime surface layer at most other sites is some tens of metres thick. For Dome C, Hammerschlag et al. (2006) have designed an extremely stiff $30 \mathrm{~m}$ tower capable of carrying a telescope of up to 100 tonnes; such a tower would be ideal for PILOT.

The alternative to a tower is to use Ground Layer Adaptive Optics (GLAO). GLAO is under active development for use on telescopes at temperate sites. At Dome C, its advantage would be even greater, as the free-atmosphere seeing that is left uncorrected by GLAO is significantly better than at those sites. It is worth noting that an adaptive secondary mirror is naturally conjugated to a point close to the telescope; in the case of a Gregorian mirror the conjugation is ideal - a few tens of metres above the telescope.

An additional advantage of GLAO is that it can also correct for "dome seeing", low spatial frequency errors in the mirror figure, and errors in collimation. As the required image quality from PILOT is several times better than for other 2 metre class telescopes, it may be that the least expensive way to achieve this is with an $\mathrm{AO}$ system. It is therefore unclear at this stage whether PILOT should be placed on a 30 metre tower, or on a simple base of a few metres height, or on a 5 metre snow hill.

\section{PILOT beyond the pathfinder phase}

PILOT is expected to have a productive working life of several years, during which time more specialised telescopes will be built to replace it in each of its key scientific areas. In the longer term, an interesting possible future role for PILOT is in the study of satellite debris. This is an increasing problem for space programs, as a collision with any one of the 100,000 objects between 1 and $10 \mathrm{~cm}$ in diameter that are currently in orbit could be catastrophic. The orbits of most objects of this size are currently unknown, and several programs are underway to improve existing databases. Dome $\mathrm{C}$ is an almost ideal location for a satellite debris survey telescope, as it is well placed to see polar-orbiting junk and the long twilight hours improve detectability.

\section{Acknowledgements}

We thank the ARENA Coordination Action and the Australian Research Council (ARC) for travel funding, and the ARC for providing financial support for this research.

\section{References}

Agabi, A., Aristidi, E., Azouit, M., Fossat, E., Martin, F., Sadibekova, T., Vernin, J. \& Ziad, A. 2006, PASP, 118, 344

Burton, M.G., Lawrence, J.S., Ashley, M.C.B., et al. 2005, PASA, 22, 199

Fowler, A.M., et al. 1998, Proc. SPIE, 3354, 1170 
Gillingham, P.R. 1991, Proc. ASA, 9, 55

Harper, D.A. 1989, in Astrophysics in Antarctica, Amer. Inst. Phys. conf. Proc. 198, 123

Hammerschlag, R.H., Bettonvil, F.C.M. \& Jaegers, A.P.L. 2006, Proc. SPIE, 6273, 627310

Herald, M. 1994, in Astrophys. \& Sp. Sci. Lib., 190, Infrared Astronomy with Arrays, the Next Generation, ed. I. McLean, 248

Lawrence, J. S., Ashley, M. C. B., Travouillon, T. \& Tokovinin, A. 2004, Nat, 431, 278

Storey, J.W.V., Angel, J.R.P., Lawrence, J.S., Hinz, P., Ashley, M.C.B. \& Burton, M.G. 2006, Proc SPIE, 6267, 62671E-1

Swain, M.R. \& Gallee, H. G. 2006, PASP, 118, 1190 